\title{
Birch bark compound combats metabolic syndrome
}

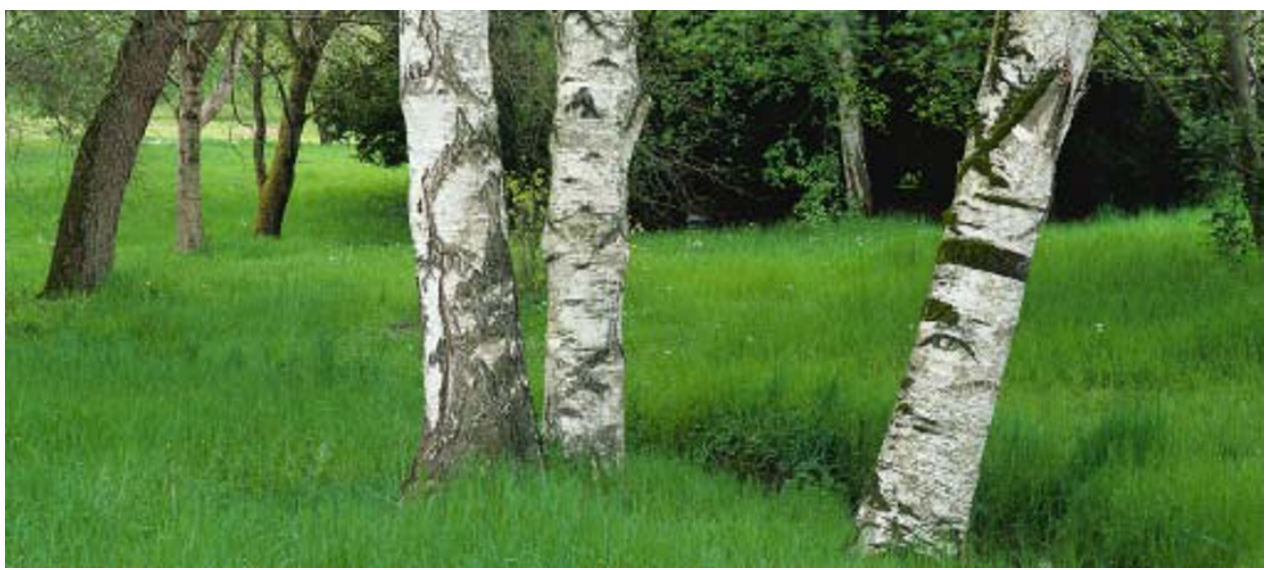

The sterol regulatory element binding protein (SREBP) family of transcription factors controls cellular lipid homeostasis by regulating the expression of genes involved in the synthesis and uptake of cholesterol, fatty acids and triglycerides. Given that hyperlipidaemia is closely associated with the development of metabolic diseases, modulating SREBP activity represents a promising therapeutic strategy. Here, Song and colleagues identify a specific small-molecule inhibitor of SREBP processing, which may be a new lead in the development of novel therapies for type 2 diabetes and atherosclerosis.

The activity of SREBPs is regulated by sterols. High levels of sterols stimulate the association between SREBP cleavage activating protein (SCAP) and insulin-induced gene 1 (INSIG1) protein, which results in retention of SREBP in the endoplasmic reticulum. Low levels of sterols, however, stimulate SCAP-mediated transport of SREBP to the Golgi complex, where it is cleaved and the mature, nuclear form of SREBP is released. Currently available inhibitors of SREBP processing cannot be used clinically as they activate the liver X receptor (LXR), which mediates cholesterol efflux but also upregulates the SREBP1c isoform, which leads to fatty acid synthesis. Song and colleagues therefore aimed to identify a specific SREBP inhibitor and investigate its potential in the treatment of metabolic diseases.

First, using chemical screening, they identified betulin as a potent inhibitor of SREBP activity. Betulin is a pentacyclic triterpene that is abundantly found in birch bark. In in vitro mechanistic studies, betulin was shown to directly bind to SCAP and stimulate its association with INSIG1 by blocking SREBP processing, downregulating genes involved in cholesterol and fatty acid biosynthesis, and decreasing cellular lipid levels. Importantly, betulin was specific for SREBPs and had no effect on the LXR.
To assess the activity of betulin in vivo and demonstrate the therapeutic potential of SREBP inhibition, mice on a Westernized diet (consisting of high fat and high cholesterol) were treated daily by gastric irrigation with either betulin or vehicle control. After 6 weeks, weight gain was lower in betulin-treated mice owing to increased energy expenditure, which suggests that betulin might prevent diet-induced obesity. In accordance with the in vitro studies, betulin decreased lipid levels in serum and tissues, and these effects were mediated through modulation of the SCAP-SREBP pathway.

Furthermore, betulin improved glucose tolerance and insulin sensitivity and lowered the elevated fasting blood glucose level seen in control mice. Moreover, the expression of genes involved in cholesterol and fatty acid synthesis was reduced, whereas white adipose tissue genes that are known to have anti-diabetic and anti-inflammatory effects were upregulated. Finally, in a mouse model of atherosclerosis, 14 weeks of betulin treatment reduced the size and improved the stability of plaques.

Notably, in each of these in vivo studies, betulin was equally or more effective than lovastatin, which is a widely prescribed anti-hypercholesterolaemic drug that acts through a different mechanism and is associated with adverse effects.

In summary, these findings support the concept of inhibiting the SREBP pathway to treat hyperlipidaemia and associated metabolic diseases, and this approach may offer advantages over existing therapeutic strategies.

Sarah Crunkhorn

ORIGINAL RESEARCH PAPER Tang, J.-J. et al. Inhibition of SREBP by a small molecule, betulin, improves hyperlipidemia and insulin resistance and reduces atherosclerotic plaques. Cell Metab. 13, 44-56 (2011) 